\title{
Iminophosphorane-mediated one-pot synthesis of 1,3,4-oxadiazole derivatives
}

\author{
Ali Ramazani*a and Ali Souldozi ${ }^{\mathrm{b}}$ \\ ${ }^{a}$ Chemistry Department, Zanjan University, P O Box 45195-313, Zanjan, Iran \\ ${ }^{b}$ Chemistry Department, Islamic Azad University-Urmia Branch, P.O. Box 969, Urmia, Iran \\ E-mail: aliramazani@gmail.com
}

\begin{abstract}
Reaction of 4-substituted benzoic acid derivatives with ( $N$-isocyanimino)-triphenylphosphorane proceeds smoothly at room temperature to afford the corresponding 2-aryl-1,3,4-oxadiazoles via an intramolecular $a z a$-Wittig reaction in excellent yields under neutral conditions. The structures of the products were deduced from their IR, ${ }^{1} \mathrm{H} \mathrm{NMR}$, and ${ }^{13} \mathrm{C} \mathrm{NMR}$ spectra, and mass spectrometry.
\end{abstract}

Keywords: ( $N$-Isocyanimino)triphenylphosphorane, intramolecular aza-Wittig reaction, 1,3,4oxadiazole, benzoic acid derivatives, iminophosphorane, isocyanide

\section{Introduction}

Isocyanide-based reactions have been known for about 80 years, with the first described in 1921 and named after its founder, Passerini. ${ }^{1,2}$ The chemistry of the isocyanides began in 1859 when Lieke prepared allyl isocyanide as the first isocyanide. ${ }^{3}$ Lieke, like many chemists today, was immediately struck by their strange repulsive odor, one of the only negatives of this branch of chemistry. The classical syntheses of isocyanides were developed in 1867 by Gautier. ${ }^{4}$ For the following century only 12 isocyanides were known and rather few types of reactions had been described. Thus for a whole century, from 1859 to 1958, isocyanides were not readily available, and the chemistry of the isocyanides remained an under-investigated part of organic chemistry. ${ }^{5}$

In 1921, Passerini pioneered the use of isocyanides and successfully developed a threecomponent synthesis of $\alpha$-acyloxycarboxamide by reaction of a carboxylic acid, an aldehyde, and an isocyanide. ${ }^{1}$ Today most isocyanide-based multi-component reactions chemistry (IMCR) relates to the classical reactions of Passerini and Ugi. Indeed, the large number of different scaffolds now available mostly builds on these two IMCRs and their combination with other types of reactions. ${ }^{6-28}$ Passerini reactions involve an oxo-component, an isocyanide, and a nucleophile. Ugi reactions are defined as the reaction of a Schiff base or an enamine with a 
nucleophile and an isocyanide, followed by a (Mumm) rearrangement reaction. The Passerini reactions are beginning to find utility in the drug discovery process, and total syntheses of biologically relevant natural products. ${ }^{22}$

Organophosphorus compounds ${ }^{29-31}$ have been extensively employed in organic synthesis as useful reagents, as well as ligands, in a number of transition metal catalysts. ${ }^{30}$ Iminophosphoranes are a special type of zwitterions, which bear a strongly nucleophilic electron rich nitrogen. The electron distribution around the $\mathrm{P}^{+}-\mathrm{N}^{-}$bond and its consequent chemical implications have been probed and assessed through theoretical, spectroscopic and crystallographic investigations. ${ }^{32-34}$ The proton affinity of these iminophosphoranes can be used as a molecular guide to assess their utility as synthetic reagents and their function as ligands in coordination and organometallic chemistry. ${ }^{32-34}$

The intramolecular version of the $a z a$-Wittig-type reaction has attracted considerable attention recently because of its high potential for the synthesis of a wide variety of nitrogen heterocycles, which can be attributed, in good measure, to the rapid progress in the preparation of functionalized iminophosphoranes. Several interesting hetero-cyclization reactions involving iminophosphoranes have been reviewed. ${ }^{32}$ These compounds can easily be converted through $a z a$-Wittig reaction with isocyanates, carbon dioxide, or carbon disulfide into functionalized hetero-cumulenes which exhibit a rich chemistry of unusual synthetic promise. ${ }^{32}$ The nucleophilicity at the nitrogen is a factor of essential mechanistic importance in the use of these iminophosphoranes as aza-Wittig reagents. Iminophosphoranes are important reagents in synthetic organic chemistry, especially in the synthesis of naturally occurring products, compounds with biological and pharmacological activity. ${ }^{32}$ However, the organic chemistry of $\left(N\right.$-isocyanimino)triphenylphosphorane 2 remains almost unexplored. ${ }^{33,34}(N-$ isocyanimino)triphenylphosphorane $\mathbf{2}$ is expected to have synthetic potential because it provides a reaction system in which the iminophosphorane group can react with a reagent having a carbonyl functionality. ${ }^{33,34}$ In recent years, we have established a one-pot method for the synthesis of organophosphorus compounds. ${ }^{35-44}$ As part of our ongoing program to develop efficient and robust methods for the preparation of heterocyclic compounds (Ali Ramazani reactions), ${ }^{35-45}$ we sought to develop a convenient preparation of 2-aryl-1,3,4-oxadiazoles 5 from 4-substituted benzoic acid derivatives $\mathbf{1}$ and ( $N$-isocyanimino)triphenylphosphorane $\mathbf{2}$ in excellent yields under neutral conditions (Scheme 1).

1,3,4-Oxadiazoles have attracted interest in medicinal chemistry as surrogates of carboxylic acids, esters, and carboxamides. ${ }^{46}$ They are an important class of heterocyclic compounds that have a wide range of pharmaceutical and biological activities including antimicrobial, antifungal, anti-inflammatory, and antihypertensive. ${ }^{46}$ Several methods have been reported in the literature for the synthesis of 1,3,4-oxadiazoles. These protocols are multi-step in nature. ${ }^{46}$ The most general method involves the cyclization of diacylhydrazides with a variety of reagents, such as thionyl chloride, phosphorus oxychloride, or sulfuric acid, usually under harsh reaction conditions. ${ }^{46}$ Few reliable and operationally simple examples have been reported for the one-step 
synthesis of 1,3,4-oxadiazoles, especially from readily available carboxylic acids and acid hydrazides. $^{46}$

\section{Results and Discussion}

In the last years, several synthetic methods have been reported for the preparation of $(N$ isocyanimino)triphenylphosphorane $\left(\mathrm{CNNPPh}_{3}\right) \mathbf{2}\left(\right.$ Scheme 1). ${ }^{33,34}$ There are several reports for the use of ( $N$-isocyanimino)triphenylphosphorane 2 in the synthesis of metal complexes. ${ }^{33,34}$ However, application of $\mathbf{2}$ in the synthesis of organic compounds has been fairly rare. ${ }^{25}$ As part of our ongoing program to develop efficient and robust methods for the preparation of heterocyclic compounds, ${ }^{35-45}$ we sought to develop a convenient preparation of 2-aryl-1,3,4oxadiazoles $\mathbf{5}$ from 4-substituted benzoic acid derivatives $\mathbf{1}$ and ( $N$-isocyanimino)triphenylphosphorane $\mathbf{2}$ in excellent yields under neutral conditions (Scheme 1).<smiles>[X]c1ccc(C(=O)O)cc1</smiles>

1

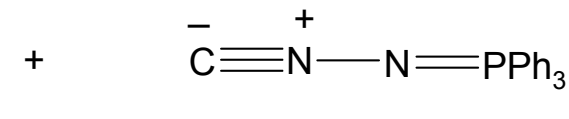

2

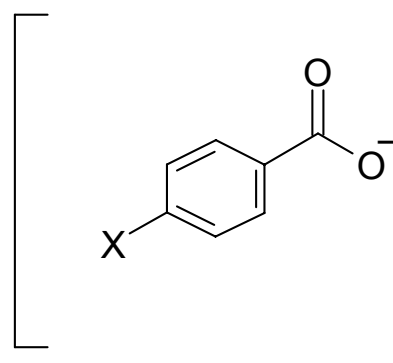<smiles>C#[N+]N=[PbH2]</smiles>

3

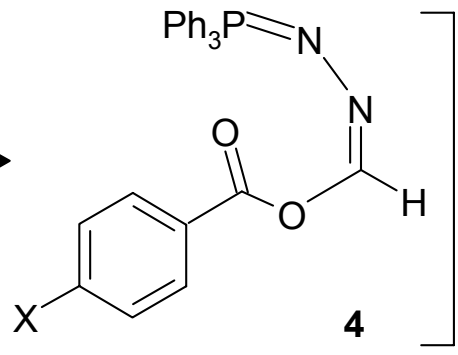<smiles>[X]c1ccc(-c2nnc(C)o2)cc1</smiles>

5

$+$<smiles>[PbH2][PbH2]</smiles>

6

1, 3, 4, $5: X=I, C N, \mathrm{CO}_{2} \mathrm{Me}, \mathrm{OAc}, \mathrm{Et}$

Scheme 1. Preparation of 2-aryl-1,3,4-oxadiazoles 5 from 4-substituted benzoic acids.

The 4-substituted benzoic acid derivatives $\mathbf{1}$ and ( $N$-isocyanimino)-triphenylphosphorane $\mathbf{2}$ in dry $\mathrm{CHCl}_{3}$ react together in a 1:1 ratio at room temperature to produce 2-aryl-1,3,4-oxadiazoles $\mathbf{5}$ 
and triphenylphosphine oxide 6 (Scheme 1). The reaction proceeds smoothly and cleanly under mild conditions. The mechanism of the reaction between the 4-substituted benzoic acid derivatives $\mathbf{1}$ and ( $N$-isocyanimino)-triphenylphosphorane $\mathbf{2}$ has not been established experimentally. However, a possible explanation is proposed in Scheme 1. On the basis of the well established chemistry of isocyanides, ${ }^{25}$ it is reasonable to assume that protonation of 2 by the 4-substituted benzoic acid derivatives 1 followed by a quenching of the cationic center by the conjugate base of the carboxylic acid can generate the iminophosphorane $4 .^{32}$ An intramolecular $a z a$-Wittig ${ }^{32}$ reaction of the iminophosphorane 4 would lead to formation of the 2-aryl-1,3,4oxadiazoles 5 and triphenylphosphine oxide 6 (Scheme 1). The structures of the products 5a-e were deduced from their IR, ${ }^{1} \mathrm{H} \mathrm{NMR}$, and ${ }^{13} \mathrm{C}$ NMR spectra. The mass spectra of these compounds displayed molecular ion peaks at the appropriate $m / z$ values. The IR spectrum of $\mathbf{5 a}$ showed strong adsorptions at $3134(\mathrm{CH}$, aromatic); 1685 and $1592(\mathrm{C}=\mathrm{C}$, aromatic); 1323 and 762 (oxadiazole and aromatic parts) $\mathrm{cm}^{-1}$ indicating the presence of the mentioned functionalities in its formula. The ${ }^{1} \mathrm{H}$ NMR spectrum of 5a compound exhibited three signals readily recognized as arising from aromatic moiety $\left[\delta=7.80\left(\mathrm{~d}, 2 \mathrm{H},{ }^{3} J_{\mathrm{HH}}=7.5 \mathrm{~Hz}\right.\right.$, arom. $), 7.88\left(\mathrm{~d}, 2 \mathrm{H},{ }^{3} J_{\mathrm{HH}}=7.5\right.$ $\mathrm{Hz}$, arom.) ] and a $\mathrm{CH}$ of oxadiazole ring $(\delta=8.49$, s, $1 \mathrm{H})$. The ${ }^{1} \mathrm{H}$ decoupled ${ }^{13} \mathrm{C}$ NMR spectrum of 5a showed six distinct resonances [ $\delta=99.04$ and 122.87 (2 C, arom.); 128.41 and 138.43 (4 $\mathrm{CH}$, arom.); 152.77 (1 CH, oxadiazole); 164.23 (1 C, oxadiazole)] that are in agreement with the formula and structure of 5a. Partial assignment of these resonances is given in the spectral analysis section (see Experimental Section). We have also used other carboxylic acids (acetic acid, phthalic acid, maleic acid, chloroacetic acid, trichloroacetic acid, benzylic acid, 3,5dinitrosalicylic acid, (2-hydroxyphenyl)acetic acid, 4-hydroxybenzoic acid, 3-hydroxybenzoic acid, salicylic acid, 4-aminobenzenesulfonic acid, 5-sulfosalicylic acid, nicotinic acid, 3methoxyphenylacetic acid, 4-methoxyphenylacetic acid, diphenylacetic acid, 4fluorophenylacetic acid, 3-phenylpropanoic acid, 4-chlorophenylacetic acid, benzenesulfonic acid, acetylenedicarboxylic acid and methyl red) in this reaction, but no products were observed after $24 \mathrm{hr}$. TLC indicated that the solution contained starting materials and some minor colored products.

In summary, we have found a new method for the preparation of 2-aryl-1,3,4-oxadiazoles $\mathbf{5}$ from 4-substituted benzoic acid derivatives $\mathbf{1}$ and ( $N$-isocyanimino)-triphenylphosphorane $\mathbf{2}$ in excellent yields under neutral conditions (Ramazani 1,3,4-oxadiazole synthesis). We believe that the reported method offers a mild and simple route for the preparation of these derivatives. Its ease of work-up and reaction conditions make it a useful addition to modern synthetic methodologies. Other aspects of this process are under investigation.

\section{Experimental Section}

General Procedures. Starting materials and solvents were obtained from Merck (Germany) and Fluka (Switzerland) and were used without further purification. The methods were used to follow 
the reactions are TLC and NMR. TLC and NMR indicated that there is no side product. Melting points were measured on an Electrothermal 9100 apparatus and are uncorrected. IR spectra were measured on a Shimadzu IR-460 spectrometer. ${ }^{1} \mathrm{H}$ and ${ }^{13} \mathrm{C}$ NMR spectra were measured $\left(\mathrm{CDCl}_{3}\right.$ solution) with a BRUKER DRX-250 AVANCE spectrometer at 250.0 and $62.5 \mathrm{MHz}$, respectively. Elemental analyses were performed using a Heraeus CHN-O-Rapid analyzer. Mass spectra were recorded on a FINNIGAN-MATT 8430 mass spectrometer operating at an ionization potential of $20 \mathrm{eV}$. ( $N$-Isocyanimino)triphenylphosphorane 2 was prepared based on a reported procedure. ${ }^{33,34}$ Flash chromatography columns were prepared from Merck silica gel powder.

\section{General procedure for the preparation of compounds 4 and 5}

To a magnetically stirred solution of ( $N$-isocyanimino)triphenylphosphorane ${ }^{33,34} \mathbf{2}(0.302 \mathrm{~g}, 1$ mmol) in dry $\mathrm{CHCl}_{3}(4 \mathrm{~mL})$ was added dropwise a solution of 4-substituted benzoic acid derivatives $1(1 \mathrm{mmol})$ in dry $\mathrm{CHCl}_{3}(4 \mathrm{~mL})$ over $15 \mathrm{~min}$. The mixture was stirred for $6 \mathrm{~h}$ at room temperature. The solvent was removed under reduced pressure and the viscous residue was purified by flash column chromatography (silica gel; petroleum ether-ethyl acetate (10:2)). The solvent was removed under reduced pressure and the product 5 was obtained. The characterization data of the compounds are given below:

2-(4-Iodophenyl)-[1,3,4]oxadiazole (5a). White crystals; m.p: $149.8-150.6{ }^{\circ} \mathrm{C}$; Yield: $84 \%$. IR $(\mathrm{KBr}): v=3134,1685,1592,1323$ and $762 \mathrm{~cm}^{-1} .{ }^{1} \mathrm{H} \mathrm{NMR}\left(\mathrm{CDCl}_{3}\right): \delta=7.80\left(\mathrm{~d}, 2 \mathrm{H},{ }^{3} J_{\mathrm{HH}}=7.5\right.$ $\mathrm{Hz}$, arom); 7.88 (d, $2 \mathrm{H},{ }^{3} \mathrm{~J}_{\mathrm{HH}}=7.5 \mathrm{~Hz}$, arom.); 8.49 (s, $1 \mathrm{H}$, oxadiazole). ${ }^{13} \mathrm{C} \mathrm{NMR}\left(\mathrm{CDCl}_{3}\right): \delta=$ 99.04 and 122.87 (2C, arom.); 128.41 and 138.43 (4 CH, arom.); 152.77 (1 CH, oxadiazole); 164.23 (1C, oxadiazole). MS: $m / z(\%) ; 272\left(\mathrm{M}^{+}, 100\right), 231$ (58), 203 (13), 182 (8), 149 (14), 105 (22), 89 ( 32), 76 (35), 50 (36). Anal. Calcd. for $\mathrm{C}_{8} \mathrm{H}_{5} \mathrm{IN}_{2} \mathrm{O}$ (272.04): C 35.32, H 1.85, N 10.30. Found: C 35.26, H 1.59, N 10.28\%.

4-[1,3,4] Oxadiazol-2-yl-benzonitrile (5b). White crystals; m.p: 139.1-140.6 ${ }^{\circ} \mathrm{C}$. Yield: $81 \%$. IR $(\mathrm{KBr}): v=3153,2923,2231,1662,1500,1285,1100$ and $846 \mathrm{~cm}^{-1} .{ }^{1} \mathrm{H} \mathrm{NMR}\left(\mathrm{CDCl}_{3}\right): \delta=7.87$ (d, $2 \mathrm{H},{ }^{3} J_{\mathrm{HH}}=8.5 \mathrm{~Hz}$, arom..), 8.25 (d, $2 \mathrm{H},{ }^{3} J_{\mathrm{HH}}=8.5 \mathrm{~Hz}$, arom.) and 8.59 (s, $1 \mathrm{H}$, oxadiazole). ${ }^{13} \mathrm{C} \mathrm{NMR}\left(\mathrm{CDCl}_{3}\right): \delta=115.57$ (1C of nitrile); 127.33 (1C, arom.); 127.59 and 132.93 (4 CH, arom.); 153.25 (1CH, oxadiazole); 163.50 (1C, oxadiazole). MS: $m / z(\%) ; 171\left(\mathrm{M}^{+}, 56\right), 130$ (72), 115 (100), 102 (63), 75 (43), 63 (32), 44 ( 55), 41 (39). Anal. Calcd. for $\mathrm{C}_{9} \mathrm{H}_{5} \mathrm{~N}_{3} \mathrm{O}$ (171.16): C 63.16, H 2.94, N 24.55. Found: C 63.04, H 2.86, N 24.52\%.

Methyl 4-[1,3,4]oxadiazol-2-yl-benzoate (5c). White crystals; m.p: 149.1-149.4 ${ }^{\circ} \mathrm{C}$; Yield: $73 \%$. IR (KBr): $v=3115 ; 2954 ; 1723 ; 1285 ; 1115$ and $723 \mathrm{~cm}^{-1} .{ }^{1} \mathrm{H} \mathrm{NMR}\left(\mathrm{CDCl}_{3}\right): \delta=3.98(\mathrm{~s}$, $\left.3 \mathrm{H}, \mathrm{CH}_{3}\right) ; 8.19$ (s, $2 \mathrm{H}$, arom.); 8.19-8.18 (m, $2 \mathrm{H}$, arom.); 8.56 (s, 1H, oxadiazole). ${ }^{13} \mathrm{C}$ NMR $\left(\mathrm{CDCl}_{3}\right): \delta=52.53\left(\mathrm{OCH}_{3}\right) ; 127.04$ (2 CH, arom.); 127.19 (1 C, arom.); 133.09 (2 CH, arom.); 153.06 (1 CH, oxadiazole); 162.25 (1 C, oxadiazole); 166.02 (1 C, ester). MS: $m / z(\%) ; 204\left(\mathrm{M}^{+}\right.$, 41), 173 (100), 90 (33), 62 (23), 50 (22). Anal. Calcd for $\mathrm{C}_{10} \mathrm{H}_{8} \mathrm{~N}_{2} \mathrm{O}_{3}$ (204.18): C 58.82, H 3.95, N 13.72. Found: C 58.71, H 3.87, N 13.70\%. 
4-[1,3,4] Oxadiazol-2-yl-phenyl acetate (5d). White crystals; m.p: $111.8-112.1{ }^{\circ} \mathrm{C}$; Yield: $79 \%$. IR (KBr): $v=3115,2931,1762,1615,1500,1377,1208,915$ and $715 \mathrm{~cm}^{-1} .{ }^{1} \mathrm{H}$ NMR $\left(\mathrm{CDCl}_{3}\right)$ : $\delta=2.33\left(\mathrm{~s}, 3 \mathrm{H}, \mathrm{CH}_{3}\right) ; 7.26\left(\mathrm{~d}, 2 \mathrm{H},{ }^{3} J_{\mathrm{HH}}=7.0 \mathrm{~Hz}\right.$, arom.); 8.09 (d, $2 \mathrm{H},{ }^{3} J_{\mathrm{HH}}=7.0 \mathrm{~Hz}$, arom.); 8.46 (s, $1 \mathrm{H}$, oxadiazole). ${ }^{13} \mathrm{C}$ NMR $\left(\mathrm{CDCl}_{3}\right): \delta=21.10\left(\mathrm{CH}, \mathrm{CH}_{3}\right) ; 121.06$ (1 C, arom.), 122.51 and 128.49 (4 CH, arom.); 152.64 (1 CH, oxadiazole); 153.49 (1 C-O, arom.); 164.10 (1 C, oxadiazole); $168.82(1 \mathrm{C}=\mathrm{O})$. MS: $m / z(\%) ; 204\left(\mathrm{M}^{+}, 14\right), 162$ (100), 121 (73), 105 (12), 78 (13) 43 (66). Anal. Calcd. for $\mathrm{C}_{10} \mathrm{H}_{8} \mathrm{~N}_{2} \mathrm{O}_{3}$ (204.18): C 58.82, H 3.95, N 13.72. Found: C 58.69, H 3.91, N 13.73 .

2-(4-Ethylphenyl)-[1,3,4] oxadiazole (5e). White crystals; m. p: 77.3-78.2 ${ }^{\circ} \mathrm{C}$. Yield: $94 \%$. IR $(\mathrm{KBr}): v=3131,2969,2931,1615,1508$ and $854 \mathrm{~cm}^{-1} .{ }^{1} \mathrm{H}$ NMR $\left(\mathrm{CDCl}_{3}\right): \delta=1.27(\mathrm{t}, 3 \mathrm{H}$, $\left.{ }^{3} J_{\mathrm{HH}}=7.5 \mathrm{~Hz}, \mathrm{CH}_{3}\right) ; 2.72$ (q, $\left.2 \mathrm{H},{ }^{3} J_{\mathrm{HH}}=7.5 \mathrm{~Hz}, \mathrm{CH}_{2}\right) ; 7.34$ (d, $2 \mathrm{H},{ }^{3} J_{\mathrm{HH}}=8.0 \mathrm{~Hz}$, arom.); 7.99 (d, $2 \mathrm{H},{ }^{3} \mathrm{~J}_{\mathrm{HH}}=8.0 \mathrm{~Hz}$, arom.); 8.47 (s, $1 \mathrm{H}$, oxadiazole). ${ }^{13} \mathrm{C} \mathrm{NMR}\left(\mathrm{CDCl}_{3}\right): \delta=15.15\left(1 \mathrm{CH}, \mathrm{CH}_{3}\right)$; 28.88 ( $\left.1 \mathrm{CH}, \mathrm{CH}_{2}\right) ; 120.90$ (1 C, arom.); 127.12 and 128.60 (4 CH, arom.); 148.74 (1 C, arom.); 152.42 (1 CH, oxadiazole); 164.85 (1 C, oxadiazole). MS: $m / z(\%) ; 174\left(\mathrm{M}^{+}, 100\right), 139(68), 133$ (40), 77 (27), 63 (11) 51 (12). $\mathrm{C}_{10} \mathrm{H}_{10} \mathrm{~N}_{2} \mathrm{O}$ (174.20): calcd. C 68.95, H 5.79, N 16.08; found $\mathrm{C}$ 69.01, H 5.71, N 16.12.

\section{Acknowledgements}

This work was supported by the "Iran National Science Foundation: INSF" via the research project number 86053.20.

\section{References}

1. (a) Passerini, M.; Simone, L. Gazz. Chim. Ital. 1921, 51, 126. (b) Passerini, M. Gazz. Chim. Ital. 1921, 51, 181.

2. Passerini, M.; Ragni, G. Gazz. Chim. Ital. 1931, 61, 964.

3. Lieke, W. Justus Liebigs Ann. Chem. 1859, 112, 316.

4. Gautier, A. Justus Liebigs Ann. Chem. 1867, 142, 289.

5. Ugi, I. Isonitrile Chemistry; Academic Press: New York, 1971.

6. Sapi, J.; Laronze, J.-Y. ARKIVOC 2004, (vii), 208.

7. Zhu, J.; Bienayme', H. Multicomponent Reactions; Wiley-VCH: Weinheim, 2005.

8. Ugi, I. Angew. Chem., Int. Ed. 1962, 1, 8.

9. Hazeri, N.; Maghsoodlou, M.T.; Habibi-Khorassani, S. M.; Ziyaadini, M.; Marandi, G.; Khandan-Barani, K.; Bijanzadeh, H. R. ARKIVOC 2007, (viii), 34.

10. Dömling, A.; Beck, B.; Herdtweck, E., Antuch, W.; Oefner, C.; Yehia, N.; Gracia-Marques, A. ARKIVOC 2007, (vxii), 99.

11. Dömling, A.; Ugi, I. Angew. Chem. Int. Ed. 2000, 39, 3168. 
12. (a) Ugi, I.; Werner, B.; Dömling, A. Molecules 2003, 8, 53. (b) Ugi, I. Pure Appl. Chem. 2001, 73, 187.

13. Dömling, A. Chem. Rev. 2006, 106, 17.

14. Banfi, L.; Guanti, G.; Riva, R.; Basso, A.; Calcagno, E. Tetrahedron Lett. 2002, 43, 4067.

15. Neo, A. G.; Delgado, J.; Polo, C.; Marcaccini, S.; Marcos, C. F. Tetrahedron Lett. 2005, 46, 23.

16. Banfi, L.; Guanti, G.; Riva, R. Chem. Commun. 2000, 985.

17. Ugi, I.; Meyr, R.; Fetzer, U.; Steinbrückner, C. Angew. Chem. 1959, 71, 386.

18. Ugi, I.; Steinbrückner, C. Angew. Chem. 1960, 72, 267.

19. Hoffmann, R. Angew. Chem., Int. Ed. 2001, 40, 3337.

20. Achatzb, S.; Dömling, A. Bioorg. Med. Chem. Lett. 2006, 16, 6360.

21. Cristau, P.; Vors, J. P.; Zhu, J. Tetrahedron Lett. 2003, 44, 5575.

22. Hulme, C.; Gore, V. Curr. Med. Chem. 2003, 10, 51.

23. Souldozi, A.; Ślepokura, K.; Lis, T.; Ramazani, A. Z. Naturforsch. 2007, 62b, 835.

24. Souldozi, A.; Ramazani, A. Tetrahedron Lett. 2007, 48, 1549.

25. Souldozi, A.; Ramazani, A.; Bouslimani N.; Welter, R. Tetrahedron Lett. 2007, 48, 2617.

26. Ahmadi, E.; Ramazani, A.; Nekomanesh Haghighi, M. Tetrahedron Lett. 2007, 48, 6954.

27. Kazemizadeh, A. R.; Ramazani, A. ARKIVOC 2008, (xv), 159.

28. Baker, R. H.; Stanonis, D. J. Am. Chem. Soc. 1951, 73, 699.

29. Ramazani, A.; Kazemizadeh, A. R.; Ahmadi, E.; Noshiranzadeh N.; Souldozi A. Curr. Org. Chem. 2008, 12, 59.

30. Kaska, W. C. Coord. Chem. Rev. 1983, 48, 1.

31. Ramazani, A.; Kazemizadeh, A. R.; Ahmadi, E.; Slepokura, K.; Lis, T. Z. Naturforsch. 2006, $61 b, 1128$.

32. Molina, P.; Vilaplana, M. J. Synthesis 1994, 1197.

33. Stolzenberg, H.; Weinberger, B.; Fehlhammer, W. P.; Pühlhofer F. G.; Weiss, R. Eur. J. Inorg. Chem. 2005, 21, 4263.

34. Chiu, T. W.; Liu, Y. H.; Chi, K. M.; Wen, Y. S.; Lu, K. L. Inorg Chem. 2005, 44, 6425.

35. Yavari, I.; Ramazani, A.; Yahya-Zadeh, A. Synth. Commun. 1996, 26, 4495.

36. Ramazani, A.; Ahmadi, E.; Kazemizadeh, A. R.; Dolatyari, L.; Noshiranzadeh, N.; Eskandari, I.; Souldozi, A. Phosphorus, Sulphur, Silicon Relat. Elem. 2005, 180, 2419.

37. Ramazani, A.; Ahmadi, E. Phosphorus, Sulfur, Silicon Relat. Elem. 2006, 181, 2725.

38. Yavari, I.; Ramazani, A. Phosphorus, Sulfur, Silicon Relat. Elem. 1997, 130, 73.

39. Ramazani, A.; Bodaghi, A. Tetrahedron Lett. 2000, 41, 567.

40. Pakravan, P.; Ramazani, A.; Noshiranzadeh, N.; Sedrpoushan, A. Phosphorus, Sulfur, Silicon Relat. Elem. 2007, 182, 545.

41. Ramazani, A.; Rahimifard, M.; Souldozi, A. Phosphorus, Sulfur, Silicon Relat. Elem. 2007, $182,1$.

42. Ramazani, A.; Rahimifard, M.; Noshiranzadeh, N.; Souldozi, A. Phosphorus, Sulfur, Silicon Relat. Elem. 2007, 182, 413. 
43. Ramazani, A.; Mohammadi-Vala, M. Phosphorus, Sulfur, Silicon Relat. Elem. 2001, 176, 223.

44. Ramazani, A.; Amini, I; Massoudi, A. Phosphorus, Sulphur, Silicon Relat. Elem., 2006, 181, 2225.

45. Ramazani, A.; Morsali, A.; Ganjeie, B.; Kazemizadeh, A. R.; Ahmadi, E.; Kempe, R.; Hertle, I. Z. Naturforsch., 2005, 60b, 569.

46. Katritzky, A. R.; Mohapatra, P. P.; Huang, L. ARKIVOC 2008, (ix), 62. 